archives-ouvertes

\title{
Integration of visual information for saccade production
}

Peggy Gerardin, Valérie Gaveau, Denis Pelisson, Claude Prablanc

\section{To cite this version:}

Peggy Gerardin, Valérie Gaveau, Denis Pelisson, Claude Prablanc. Integration of visual information for saccade production. Human Movement Science, Elsevier, 2011, 30 (6), pp.1009-1021. 10.1016/j.humov.2011.01.004 . hal-02196719

\section{HAL Id: hal-02196719 \\ https://hal.archives-ouvertes.fr/hal-02196719}

Submitted on 29 Jul 2019

HAL is a multi-disciplinary open access archive for the deposit and dissemination of scientific research documents, whether they are published or not. The documents may come from teaching and research institutions in France or abroad, or from public or private research centers.
L'archive ouverte pluridisciplinaire HAL, est destinée au dépôt et à la diffusion de documents scientifiques de niveau recherche, publiés ou non, émanant des établissements d'enseignement et de recherche français ou étrangers, des laboratoires publics ou privés. 


\title{
Integration of visual information for saccade production
}

\author{
Peggy Gerardin*, Valérie Gaveau, Denis Pélisson, Claude Prablanc \\ Lyon Neuroscience Research Center, INSERM U1028, CNRS UMR5292, ImpAct Team, Bron, France \\ Université Lyon 1, Villeurbanne, France
}

\section{A R T I C L E I N F O}

\section{Article history:}

Available online $\mathrm{xxxx}$

\section{Psyc INFO classification:}

2330

\section{Keywords:}

Reactive saccades

Corrective saccades

Visual integration

Visual context

\begin{abstract}
A B S T R A C T
To foveate a visual target, subjects usually execute a primary hypometric saccade (S1) bringing the target in perifoveal vision, followed by a corrective saccade (S2) or by more than one S2. It is still debated to what extent these S2 are pre-programmed or dependent only on post-saccadic retinal error. To answer this question, we used a visually-triggered saccade task in which target position and target visibility were manipulated. In one-third of the trials, the target was slightly displaced at S1 onset (so-called double step paradigm) and was maintained until the end of S1, until the start of the first S2 or until the end of the trial. Experiments took place in two visual environments: in the dark and in a dimly lit room with a visible random square background. The results showed that S2 were less accurate for shortest target durations. The duration of post-saccadic visual integration thus appears as the main factor responsible for corrective saccade accuracy. We also found that the visual context modulates primary saccade accuracy, especially for the most hypometric subjects. These findings suggest that the saccadic system is sensitive to the visual properties of the environment and uses different strategies to maintain final gaze accuracy.
\end{abstract}

\section{Introduction}

Scrutinizing a scene requires numerous fast eye movements, called saccades, which shift the retinal image of objects of interest to the fovea for detailed visual analysis. When an object is located in the

* Corresponding author at: INSERM ImpAct Team, 16 av. du Doyen Lépine, 69676 Bron Cedex, France. Tel.: +33 (0)4 729134 35; fax: +33 (0)4 72913401 .

E-mail address: peggy.gerardin@inserm.fr (P. Gerardin).

0 
periphery of our visual field, a main saccade and (at least) a corrective one are needed to foveate it. Corrective saccades have a shorter latency than the main saccade and an amplitude of about $10 \%$ of the main saccade. The process underlying corrective saccade production is still debated in terms of its possible dependency on retinal feedback (Becker, 1976; Becker \& Fuchs, 1969; Deubel, Wolf, \& Hauske, 1982; Henson, 1978; Kapoula, Robinson, \& Hain, 1986; Prablanc \& Jeannerod, 1975; Prablanc, Masse, \& Echallier, 1978). In other words, can corrective saccades be pre-programmed or do they solely depend on a retinal error signal encoded after the end of the primary saccade?

Corrective saccades have been explored by varying the time and/or location of re-appearance of the initial target. Prablanc and Jeannerod $(1974,1975)$ found that corrective saccades were generally absent when the visual input was turned off before the onset of the main (or primary) saccade. However, when a brief visual input was restored after saccade completion corrective saccades were elicited. Latencies and amplitudes varied according to the extent of the initial error of the primary saccade. These results seemed to contradict a previous hypothesis stating that corrective saccades were preprogrammed since their latencies were too short to allow a new computation of the target location (Becker, 1976; Becker \& Fuchs, 1969). However the contradiction was only apparent as it was further shown that when primary saccades were very hypometric (gain $<0.8$ ) secondary saccades were systematically elicited without the need of a new retinal input, and that the probability of occurrence of such internally triggered saccades decreased as the primary saccade gain increased (Prablanc et al., 1978). Deubel et al. (1982) contested Becker and Fuchs' findings in showing that corrective saccades can only occur when a stepped second target is switched on (as opposed to when no second target arises). The authors varied the time of reappearance of the target (0 ms - no target reappearance to $60 \mathrm{~ms}$ after the primary saccade). They found that no corrective saccade is generated until the target reappearance, showing that retinal feedback is inherent to their generation. Other results, however, showed the retinal processing of the error during the late phase of the saccade to elicit a secondary saccade, although not fully corrective (Prablanc et al., 1978).

Eggert, Ditterich, and Straube (1999) also investigated the influence on corrective saccades of a secondary target (stepped backward or forward) occurring at different times during the primary saccade. They found that when this second target appeared during the deceleration phase of the primary saccade, the latency of corrective saccades was significantly longer than when the secondary target appeared during the acceleration phase of the primary saccade. This observation was replicated in an eye-hand coordination task where subjects had to look and point at a peripheral visual target (Gaveau et al., 2008). This suggests that corrective saccades result from a processing of the retinal signal and that intra-saccadic visual stimulus directly affects the timing of the corrective saccade, despite the well-known elevation of the visual threshold during execution of the saccade (Greenhouse \& Cohn, 1991; MacAskill, Jones, \& Anderson, 2003; Matin, 1974).

In most studies in the literature, the background surrounding visual targets provides no visual information, and is generally uniform or black. However, the visual environment we live in is much more complex. The role of the visual context on corrective saccades has not yet been explored. Some studies have looked at the influence of a visual background on the primary saccade, especially on saccadic adaptation (when the amplitude of the primary saccade is progressively adjusted to a systematic perturbation of target position). Ditterich, Eggert, and Straube (2000) have shown that introducing an intrasaccadic displacement of a visual background (white random dot pattern on a black screen) influences saccadic adaptation when a large target is used. According to the authors, the target size contributes to the size of the subjects' focus of attention. When its size is large enough with respect to the items of the visual background, the numerous elements of the background which fall within the attention focus are processed by the visual system. Thus, under these conditions, an intrasaccadic shift of the background can generate an error signal. Another study has shown that the visual attribute of a target could be an efficient cue to induce selective saccadic adaptation (Herman, Harwood, \& Wallman, 2009). In the same session, subjects could adapt their saccades to one type of target (e.g., flickering illuminated circle) that stepped back intra-saccadically, but did not adapt saccades to another target (e.g., non-flickering illuminated circle) that remained stationary. In addition, in a control experiment, the observation of similarly adapted saccades to both types of target when the stationary target was switched off intra-saccadically suggests that processing of the visual attribute of the target as a contextual cue required post-saccadic feedback. However, these experiments have again used light 
spots on dark background. White, Stritzke and Gegenfurtner (2008) have recently studied the effect of natural scenes on saccadic behavior. They found that saccade latencies were faster in the context of natural backgrounds as compared to unstructured backgrounds. All these studies suggest a possible influence of the visual context on primary saccades gain and latency, but none have addressed a possible effect on the performance of corrective saccades.

In the present study, we investigated the temporal and spatial properties of the visual stimulus required to elicit corrective saccades. The range of target amplitudes was large enough to favor the generation of secondary saccades, and targets could be randomly stepped either backward or forward in some trials. The possible effect of visual background on corrective saccade production was also tested.

Our results show that short intra-saccadic target presentations are sufficient to elicit secondary saccades, although not fully corrective, suggesting an intrasaccadic retinal error signal processing. They also show that, for the most hypometric subjects, the primary saccade gain is increased by the presence of a visual background and consequently, non-retinal secondary saccades which are frequent in these subjects, tend to be suppressed.

\section{Methods}

\subsection{Observers}

Six subjects (mean age $\pm S D$ : $32 \pm 8$ ) participated in this study. All observers had normal or corrected to normal vision and gave written informed consent. Experiments were conducted in accordance with the Declaration of Helsinki and under the terms of local legislation.

\subsection{Experimental set up and stimuli}

Experiments took place in a dark room (Dark Background experiment) or dimly lit room with a random square pattern $(126 \times 60 \mathrm{~cm})$ as a background (Visual Background experiment). Subjects sat in front of a $45^{\circ}$ slanted table (see Fig. 1a). Head position and orientation were controlled with a chin rest and head restraint. An array of light-emitting diodes (LEDs, $3 \mathrm{~mm}$ diameter) located above a half reflecting mirror provided visual targets seen on the table surface, along a fronto-parallel line $56 \mathrm{~cm}$ from the eyes. Subjects saw the targets superimposed on a dark or visible background according to experiments. They were instructed to first look at a fixation target (located at $10^{\circ}$ left from the sagittal plane) and then to shift their eyes as soon as the fixation target was replaced by a randomly presented target (three possible target locations: $12.5^{\circ}, 20^{\circ}$ or $27.5^{\circ}$ in the right hemifield; Fig. $1 \mathrm{~b}$ ). Thus, the ocular angular displacement necessary to reach the target (target retinal eccentricity) was respectively $22.5^{\circ}, 30^{\circ}$ or $37.5^{\circ}$.

Horizontal eye movements were recorded with an Eye-link2 (SR Research, Canada) at a $500 \mathrm{~Hz}$ frequency. Eye position signals were processed on-line (differentiation with a two-point central difference derivative algorithm with a sampling interval of $1 / 500$ and step size of $4 \mathrm{~ms}$ (Bahill \& McDonald, 1983)), allowing to detect the onset and end of primary and secondary saccades based on a velocity threshold (where the velocity exceeded $30^{\circ} / \mathrm{s}$ ) and to trigger visual events (target off or target jump), as detailed below. The complete loop of processing between eye position recording and visual display changes was short, with a maximum delay of $4 \mathrm{~ms}$.

\subsection{Design and procedure}

Each subject performed two experiments (Dark Background and Visual Background) in a counterbalanced order.

In the first experiment, each subject performed 200 trials in complete darkness (Dark Background); the experiment was subdivided in 2 blocks of randomized trials.

Targets could either be displaced (Jump condition, $36 \%$ of trials) or remain fixed at their original position (Fix condition, 64\% of trials). In the Jump condition, the double-step paradigm was used to modify the error of the primary saccade: the initial target at $20^{\circ}$ was switched to a $12.5^{\circ}$ target 
a

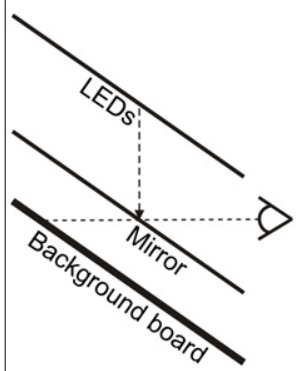

C

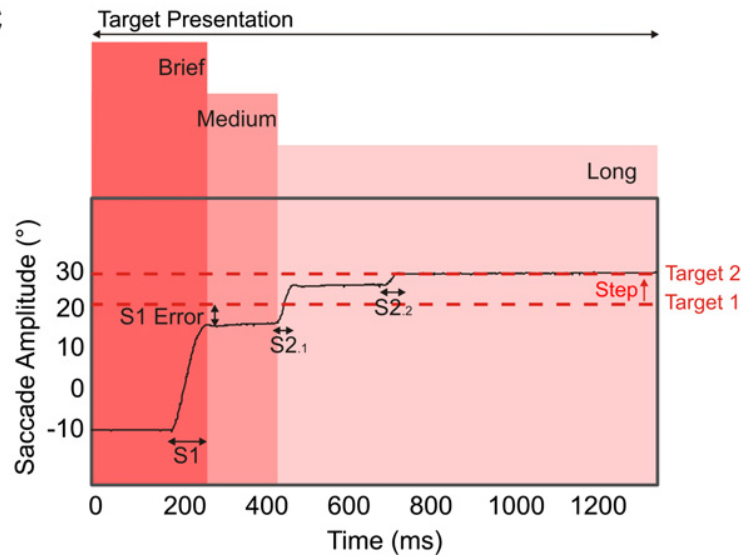

Fig. 1. Experimental set-up and procedure. (a) Side view: the LED targets were seen through a half reflecting mirror so that they appeared to the subject on the background board. (b) Locations of the LED targets with respect to the subject's trunk. (c) Duration of target jump. Individual saccadic trace (black) for a target jumping from 30 to $37.5^{\circ}$ at $\mathrm{S} 1$ saccade onset. Target jump could be brief (turned off at S1 end), medium (turned off at S2.1 saccade onset) or long (target visible until end of trial). Note that depending on subjects (hypometric subjects), additional S2 (S2.2) could be performed.

(Backward condition) or to a $27.5^{\circ}$ target (Forward condition) at saccade onset. Among these Jump trials, the timing of target presentation was randomly varied to investigate the visual target information processing: the target was maintained either until the end of the primary saccade S1 (Brief timing), until the start of the first secondary saccade S2.1 (Medium timing), or until the end of the trial (Long timing) (see Fig. 1c). All conditions described above were randomized to avoid subject's anticipation or any saccadic adaptation.

A second experiment replicated the first one with an added Visual Background (a square wave random pattern (made of $3 \mathrm{~mm}$ black and white squares) filling the entire experimental field) in order to determine the visual requirements for corrective saccade production by decreasing the detectability of the target and the intrasaccadic target jump ${ }^{1}$. All conditions cited above are summarized in Table 1.

\subsection{Off-line data analysis}

The horizontal eye position signal was first filtered (finite impulse response filter FIR, $50 \mathrm{~Hz}$ cut-off frequency) and eye velocity was computed from the filtered position signal. The sequence of eye

\footnotetext{
${ }^{1}$ The background was applied on the dimly lit device panel (as opposed to the first experiment, with a dark panel in a completely dark room). All subjects indicated that all targets (including intrasaccadic target jumps) were more difficult to detect in this condition.
} 
Table 1

Summary of all conditions used in the study. The same conditions are used for Dark and Visual Backgrounds.

\begin{tabular}{lllll}
\hline & & Fix & Backward jump & Forward jump \\
\hline Dark background & Target duration & Long & Long, medium, brief & \\
& Target eccentricity & $22.5^{\circ}, 30^{\circ}, 37.5^{\circ}$ & $30^{\circ} \rightarrow 22.5^{\circ}$ & $30^{\circ} \rightarrow 37.5^{\circ}$ \\
Visual background & Target duration & Long & Long, medium, brief & $30^{\circ} \rightarrow 37.5^{\circ}$ \\
& Target eccentricity & $22.5^{\circ}, 30^{\circ}, 37.5^{\circ}$ & $30^{\circ} \rightarrow 22.5^{\circ}$ & 3 \\
\hline
\end{tabular}

movements was defined by detecting automatically the beginning and the end of the primary and corrective saccades based on a $30 \% \mathrm{~s}$ velocity threshold. The results of this automatic procedure were then inspected and corrected manually, if necessary. Several saccade-related parameters were computed: for Fix condition, the primary saccade S1 error and the first secondary saccade S2.1 error were calculated as the difference between target position and eye position at the termination of S1 and S2.1, respectively. For Jump condition, the final target location (Target 2) was used to compute the saccadic errors for S2.1, whereas for S1 two different error parameters relative to initial and final target locations were computed (S1 error relative to Target 1 and to Target 2, respectively). For all conditions, S1 reaction time (RT) relative to target onset, S2.1 reaction time relative to S1 offset and the number of S2 were also evaluated. Final eye position error and the corresponding time of final capture were computed at the end of the last S2. Finally, the effect of each condition on S1 and S2.1 accuracy (timing of target presentation, jump or fix condition, and visual background) was assessed with separate repeated-measures ANOVA carried out on all subjects (Statistica v. 8.0, StatSoft).

\section{Results}

\subsection{General overview}

As a control condition, targets remained stationary at their original position (Fix condition). As expected, Fix condition leads to the best accuracy of final eye position (mean final error $=-0.09^{\circ}, \pm 0.05$ ) as compared to Jump condition (when targets are displaced) (Fig. 2a), with shortest S2.1 reaction time (Fig. 2b). No significant difference was observed for S1 error relative to the original target (Target 1 ) as compared to Jump condition $(F(1,5)=0.43, p=.7$, Fig. 3a). The final error was independent of the background (Visual or Dark, $F(1,5)=0.56, p=.48$, and Fig. 3b, left column). However, a significant difference for S1 accuracy was found between both backgrounds $(F(1,5)=9.5, p<.05$; Fig. 3a), as well as for final capture time (saccade completion, $F(1,5)=8, p<.05$; Fig. 3c) and the mean S2 number $(F(1,5)=7.3, p<.05$; Fig. $4 a)$.

In general, a significant interaction appeared between Jump and Target duration conditions for the final error, $F(2,10)=81, p<.0001$, but not for time of final capture, nor for the mean $S 2$ number and the S2.1 reaction time $(F(2,10)=3.3, p=.7 ; F(2,10)=3.6, p=.06 ; F(2,10)=1.3, p=.3$, respectively $)$. A strong significant interaction has been observed between Background and Jump conditions for all eye parameters (final error $F(1,5)=21, p<.005$; capture time $F(1,5)=7.5, p<.05$; S2 number $F(1$, $5)=10.9, p<.05$; S2.1 reaction time $F(1,5)=8.5, p<.05$; Figs. 3 and 4 (medium and right columns)). However, the Background condition interacted with Target duration only for final error, $F(2,10)=31.3$, $p<.001$. Detailed analyses are found in the following paragraphs.

\subsection{Jump condition}

\subsubsection{Target duration effect}

In both Dark and Visual Background experiments, each new target position (after target jump) was either lit until the end of the primary saccade S1 (Brief timing), until the start of the first secondary saccade S2 (Medium timing), or until the end of the trial (Long timing). A main effect of target duration was found for final error and for corresponding time of final capture, $F(2,10)=45.8, p<.0001$, and $F(2$, $10)=11.9, p<.005$, respectively. Long and Medium timings revealed similar final error $\left(-0.41^{\circ}, \pm 0.1\right.$ 

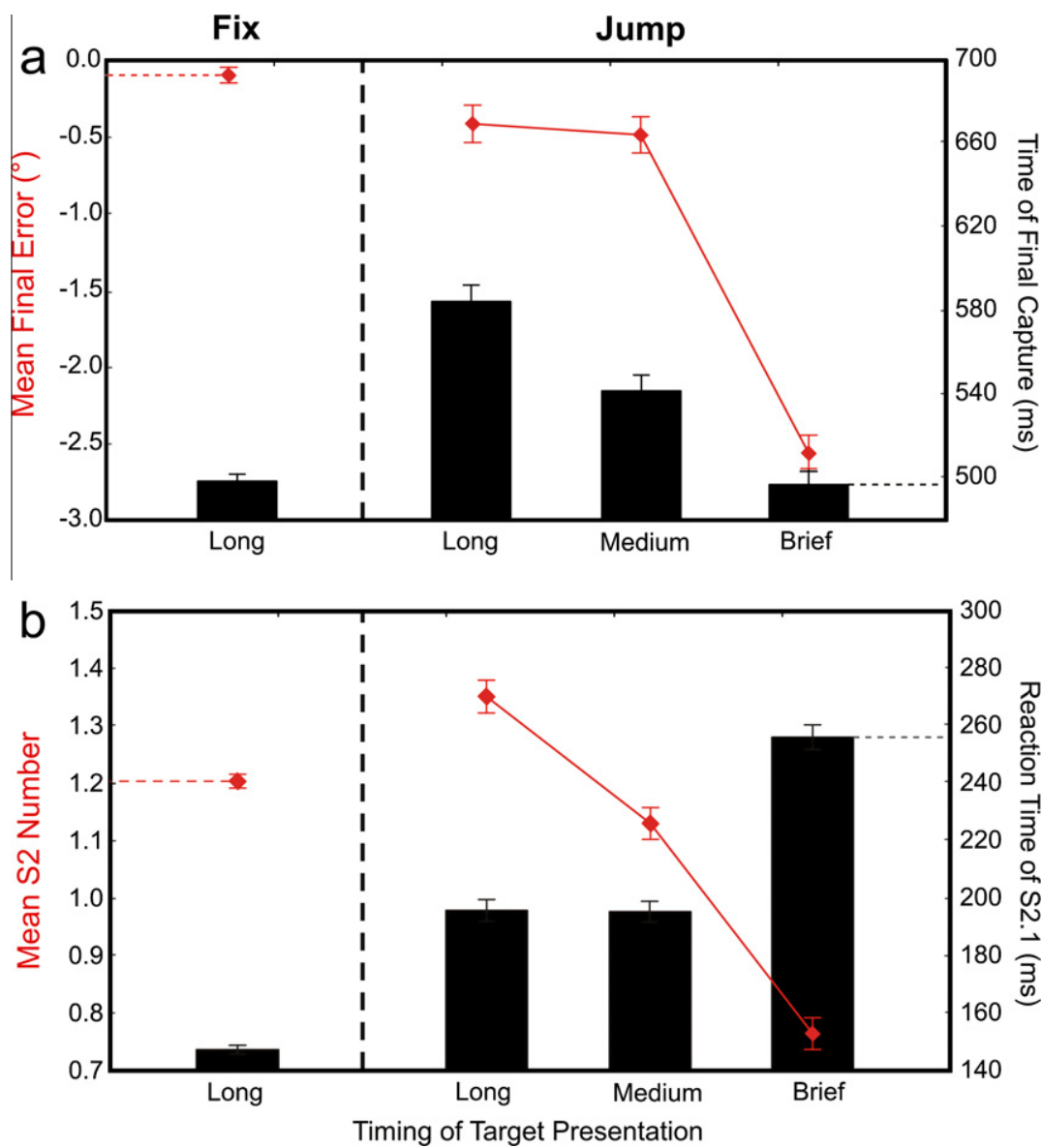

Fig. 2. Effect of target jump and of timing of target presentation on saccadic performance. Data pooled over all subjects and target eccentricities. For the Jump condition (right panel), the responses for the three timings (Long, Medium and Brief) are plotted separately. (a) Saccadic final error in degrees $(M \pm S D$, red diamonds, left scale) and time of saccadic final capture in ms ( $M \pm S D$, bars, right scale). (b) Total number of S2 per trial $(M \pm S D$, red diamonds, left scale) and reaction time of the first $S 2$ (S2.1) in $\mathrm{ms}(M \pm S D$, bars, right scale).

and $-0.48^{\circ}, \pm 0.1$, respectively) (Fig. 2a), whereas Brief timing showed the worst accuracy of all conditions $\left(-2.6^{\circ}, \pm 0.2\right)$.

The time of final capture for Long timing increases compared to Medium timing ( $584 \mathrm{~ms}, \pm 7.8$ and $541 \mathrm{~ms}, \pm 7.5$, respectively): this effect can be accounted for by the number of S2 in Long timing (Fig. $2 \mathrm{~b}$ ) that is higher than in Medium and Brief timings $(1.3, \pm 0.02,1.1, \pm 0.02$ and $0.7, \pm 0.02$ respectively). Interestingly, Medium timing lead to similar final eye position accuracy as compared with Long timing but quicker, e.g., with less S2 number. For Brief timing, final eye position inaccuracy was paired with a longer S2.1 reaction time and a lower number of corrective saccades. These differences are indeed revealed by a main effect of target duration on both S2 number and S2.1 latency, $F(2,10)=72.2$, $p<.0001$, and $F(2,10)=39.9, p<.0001$, respectively. A main effect of duration was also found for S2.1 accuracy, $F(2,10)=11.6, p<.001$. As expected, no significant effect was observed on the primary saccade $\mathrm{S} 1$ accuracy (S1 error; $F(2,10)=0.06, p=.94$ ) and latency $(F(1,5)=0.4, p=.67)$; Fig. 3a).

Thus, the jumped target has to be present at least during the inter-saccadic delay between $\mathrm{S} 1$ and S2.1 in order to be accurately foveated. When absent during the inter-saccadic delay (i.e., turned off at the end of S1), final eye position accuracy decreases. 
a
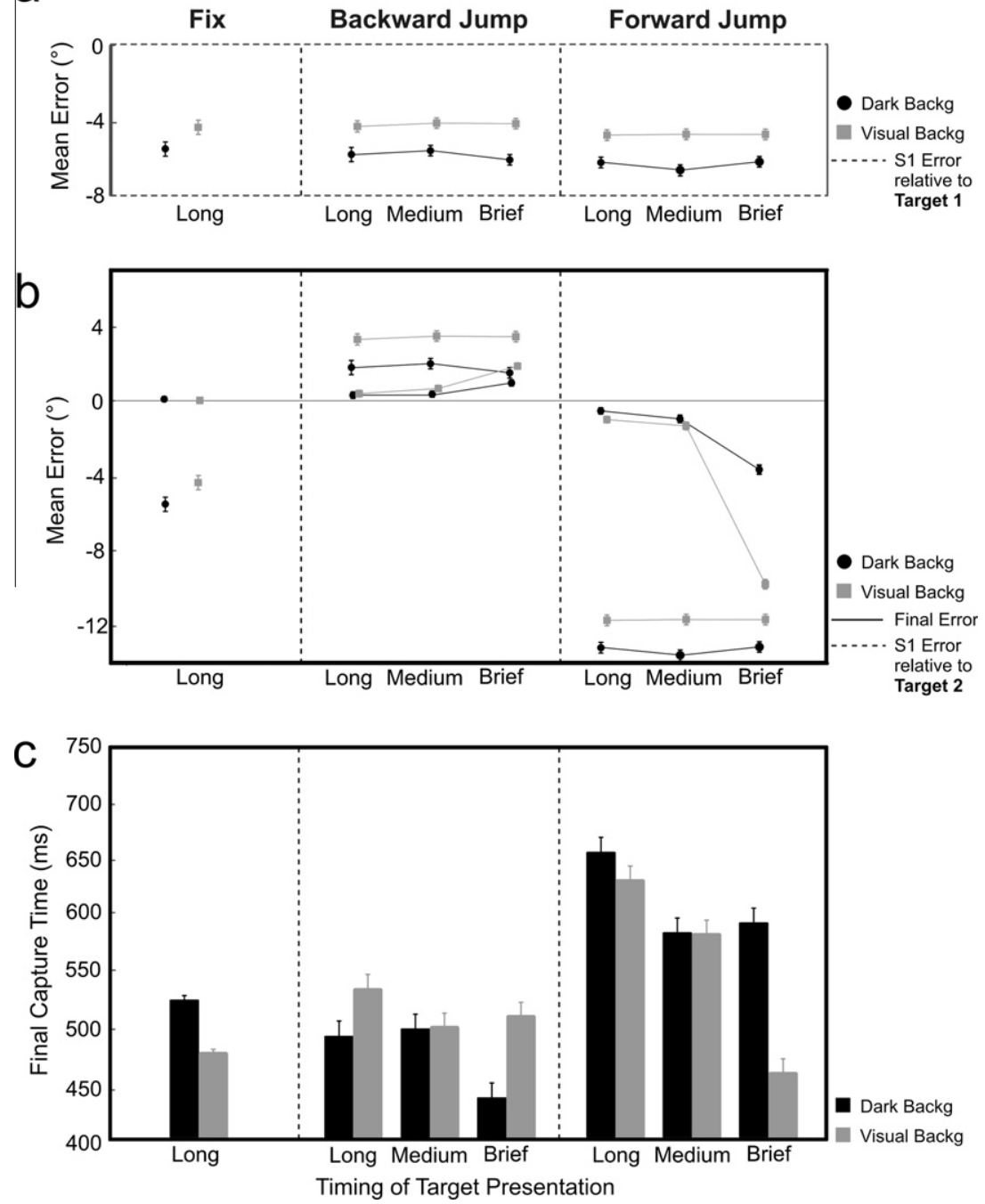

Fig. 3. Effect on $\mathrm{S} 1$ and final saccadic performances of Jump condition (Fix (no jump), Backward and Forward jumps), of timing of target presentation (Long, Medium and Brief timings) and of background (Dark or Visual Background). Data pooled over all subjects and target eccentricities. (a) S1 error relative to target 1 in degrees (Mean \pm SD) for Dark Background (Filled dark circles) and Visual Background (Filled gray squares). (b) S1 error relative to target 2 (dashed lines) and final error (solid lines) in degrees (Mean $\pm S D$ ) plotted for Dark Background (Filled dark circles) and Visual Background (Filled gray squares). (c) Time of final capture in ms (Mean $\pm S D$ ) for Dark Background (Dark bars) and Visual Background (Gray bars).

\subsubsection{Jump effect}

Targets could either jump on the left (Backward jump) or on the right (Forward jump). A main effect of this jump direction factor was found for final error, $F(1,5)=175.6, p<.0001$, and for final capture time, $F(1,5)=35.2, p<.005$. As shown in Fig. $3 \mathrm{~b}$, mean final error is close to $0^{\circ}$ in Backward condition $\left(0.57^{\circ}, \pm 0.2\right.$, center column $)$ while it increases in Forward condition $\left(-2.88^{\circ}, \pm 0.5\right.$, right column $)$. This mean difference is especially clear in 'Brief' trials, as illustrated by a significant interaction between the jump direction and the target duration factors, $F(2,10)=81.9, p<.0001$. No interaction was found for the time of final capture $(F(1,5)=3.4, p=.08$, see also Fig. $3 c)$ ). The difference of final error between Backward and Forward is essentially due to S1 error as primary saccades are generally hypometric. 

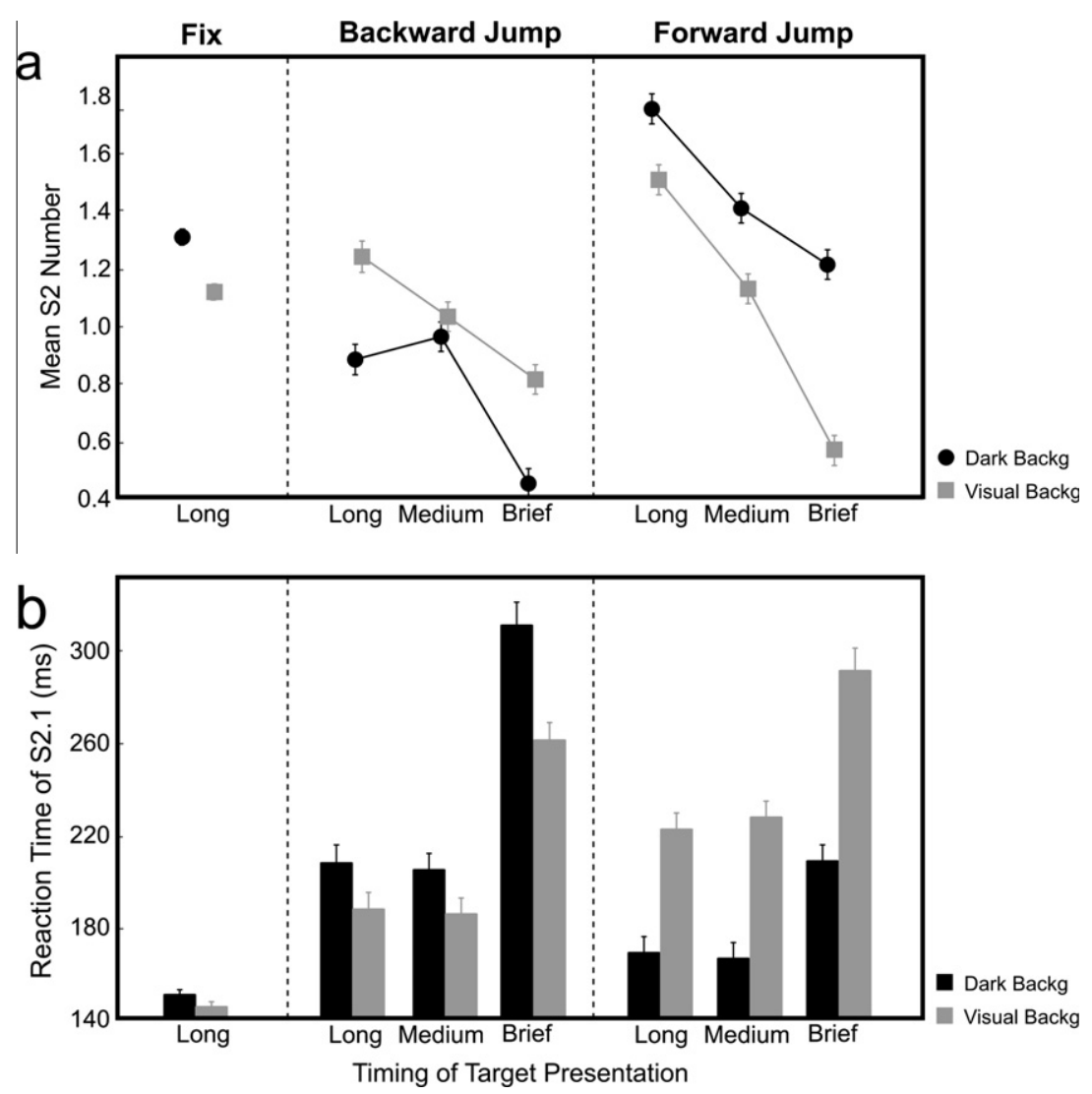

Fig. 4. Effect on S2 performances of Jump condition (Fix (no jump), Backward and Forward jumps), of timing of target presentation (Long, Medium and Brief timings) and of background (Dark or Visual Background). Data pooled over all subjects and target eccentricities. (a) Total number of secondary saccades (S2) per trial (Mean \pm SD) for Dark Background (Filled dark circles) and Visual Background (Filled gray squares). (b) First secondary saccade (S2.1) reaction time in ms (Mean $\pm S D$ ) for Dark Background (Dark bars) and Visual Background (Gray bars).

Thus, S1 error relative to target 2 (S1/T2) is smaller in Backward condition as compared to Forward condition (see Fig. 3b, medium versus right panel). This weaker S1/T2 error in Backward condition also entails less corrective saccades (see below).

Final error significantly increased for Brief timing in Backward condition $\left(0.7^{\circ}, \pm 0.15\right)$, and dramatically decreased in Forward condition $\left(-6.5^{\circ} \pm 0.25\right)$. A significant difference in time of final capture was found between Backward and Forward conditions ( $498 \mathrm{~ms} \pm 47$ and $591 \mathrm{~ms} \pm$ 40, respectively) that can be mainly explained by the number of $\mathrm{S} 2$ needed to complete the response (Fig. 4a). Indeed, a main effect of jump was found for the number of $S 2, F(1,5)=24.3, p<.005$, that significantly increased in Forward condition compared with Backward condition (1.25 \pm 0.15 and $0.9 \pm 0.1$, respectively). A main effect of jump was also found for S2.1 error, $F(1,5)=59.2, p<.001$, in Backward condition, $93 \%$ of S2.1 was defined as corrective (i.e., generated toward the target location) compared to $63 \%$ in Forward condition. This difference is essentially due to the fact that hypometric S1 brought the eyes closer to the Backward target, as explained above. No significant main effect of jump was observed for S2.1 latencies $(F(1,5)=0.48, p=.52)$.

\subsubsection{Effect of background}

One experiment took place in a completely dark environment (Dark Background), whereas the other experiment was performed in presence of a random-squares background (Visual Background). A main 
effect of the Background condition was found for S1/T1 error, $F(1,5)=87.7, p<.05$, and for final error $F(1,5)=17.3, p<.01$. This effect can be observed in Fig. 3a, where S1/T1 errors for Visual condition are systematically lower as compared with Dark condition. Considering now S1 error relative to the (jumped) target 2, the effect of the Visual Background is detrimental in Backward condition but beneficial in Forward condition. Here again this difference between Backward and Forward may be explained by S1 natural hypometry, which appeared to be slightly reduced by the visual background relative to the dark environment. No significant effect was found for other saccadic parameters (time of final capture, S2 number, S2.1 latency and S1 latency).

The Background condition also interacts with target duration, $F(2,10)=31.3, p<.0001$; final error increases with Brief timing, $F(2,10)=21.3, p<.01$, and further increases for Visual Background $\left(-3.6^{\circ}\right.$, \pm 0.5 and $-9.2^{\circ}, \pm 0.8$, respectively; Fig. $3 \mathrm{~b}$ middle and right panels).

A significant interaction between Background and Jump conditions was found for time of final capture, $F(1,5)=7.5, p<.05$ (Fig. 3c). This interaction could be explained by the number of S2 (Fig. 4a) that is significantly lower for Dark Background in Backward condition and higher in Forward condition, $F(1,5)=10.9, p<.05$. More precisely, the number of corrective saccades S2.1 slightly decreases according to the background for Backward condition (96\% in Dark, $90 \%$ in Visual condition) while it dramatically increases in Forward condition (56\% in Dark, 71\% in Visual condition). A significant effect of the background was also found for all jump conditions for S2.1 error, $F(1,5)=19.8, p<.001$ (75\% in Dark condition, $82 \%$ in Visual condition). Note that a significant interaction between Jump and Background was also found for S2.1 latencies, $F(1,5)=8.5, p<.05$ (Fig. 4b).

These effects show again that, as seen in Brief timing, final error could largely increase, and more so when visual target position can be hardly extracted due to the presence of the random-squares background (under Visual Background).

\subsection{Fix condition}

\subsubsection{Effect of background and target position}

A main effect of target eccentricity $\left(22.5^{\circ}, 30^{\circ}\right.$ or $\left.37^{\circ}\right)$ was found on final eye position accuracy, $F(2$, $10)=5.6, p<.05$. Surprisingly, an effect of background was found for time of final capture, $F(1$, $5)=21.9, p<.01$. Indeed, Dark Background led to a longer final capture compared to Visual Background: this difference in time was due to an increase of $S 2$ number $(1.35, \pm 0.016$ in Dark, versus $1.1, \pm 0.016$ for Visual condition, Fig. 4a). An effect of the background and target position was also found for S2.1 error, $F(2,10)=4, p<.01$. Corrective S2.1 were less frequent (70\%) in Dark condition than in Visual condition (87\%). As S1 endpoints were farther from target positions in Dark than in Visual condition, additional S2 had to be performed.

For the Dark condition, the number of S2 varied between subjects, $F(10,1508)=57.9, p<.0001$ (one-way between subjects ANOVA) and was correlated with their S1 hypometry. This can be seen in Fig. 5a and $\mathrm{c}$ where subjects are ranked from the most hypometric (VG) to the less one (LU). For comparable final eye position accuracies, highly hypometric subjects (VG, OS) often executed more than one S2 with short S2.1 latencies (Fig. 5b(up) and c), while the less hypometric subjects (such as $\mathrm{LJ}$ or $\mathrm{LU}$ ) rarely produced more than one S2, and S2.1 latencies were long. Surprisingly, when a Visual Background was introduced, subjects' behavior tended to be more homogeneous, with a reduction of S2 numbers for hypometric subjects (especially for target eccentricities at $22.5^{\circ}$ and $30^{\circ}$, Fig. $5 \mathrm{c}$ ), an increased of S2.1 latencies (Fig. 5b(down)) and a decreased S2.1 error for subjects VG and OS at all target eccentricities, as well as PE at $30^{\circ}$ of eccentricity. S1 accuracy varied according to subjects and Background condition, $F(5,1502)=9, p<.001$, with a decreased error for target eccentricities at $30^{\circ}$ and $37.5^{\circ}$ in Visual condition (Fig. 5a) for all subjects except VG at $37.5^{\circ}$. Finally, no effect of Background nor Target eccentricity was found for S1 latencies, $F(1,5)=0.13, p=.72$, and $F(2,10)=8$, $p=.07$, respectively.

\section{Discussion}

Our results have shown that the presence of the target immediately after S1 completion has a major effect on final error. 

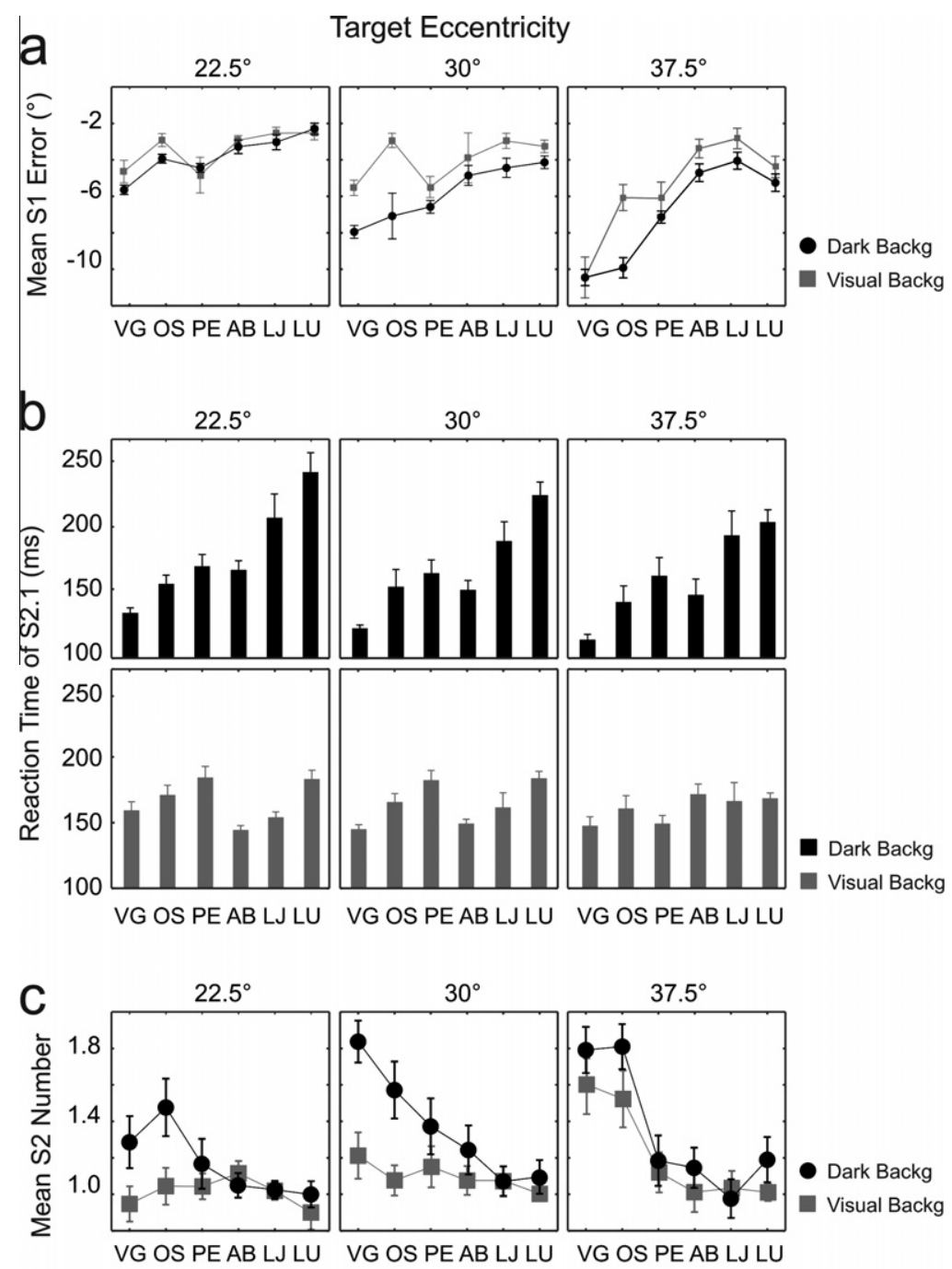

Subjects $(\mathrm{N}=6)$

Fig. 5. S1 and S2 saccadic performances for Fixed condition and for each target eccentricity (Left column: $22.5^{\circ}$, middle column: $30^{\circ}$ and right column: $37.5^{\circ}$ ) plotted separately for each subject (subjects ordered from the most to the less hypometric). (a) Primary saccades (S1) error in degrees (Mean \pm SD) for Dark Background (Filled circles) and Visual Background (Filled squares). (b) First secondary saccade (S2.1) reaction time in ms (Mean $\pm S D$ ). Results for Dark Background (upper panel) and for Visual Background (lower panel). (c) Total number of secondary saccades (S2) per trial (Mean $\pm S D$ ).

First, the increased final eye accuracy in the medium target condition, as compared to the brief condition, is related to an increased number of secondary saccades S2. A faster initiation (shorter latency) of the first secondary saccade S2.1 was also observed in the Medium timing condition. These results confirm other studies suggesting a major role of visual feedback in the production of accurate secondary saccade (Eggert et al., 1999; Munuera, Morel, Duhamel, \& Deneve, 2009; Prablanc et al., 1978).

Second, the trend toward hypometry of primary saccade S1 largely explains the various effects of Backward and Forward conditions. Indeed, the S1 hypometric error was increased by the target jump in the Forward condition, whereas it was decreased and even inverted in the Backward condition. 
Third, the introduction of a noise in the Visual Background resulted in an increased gain of the primary saccade $S 1$ (and therefore a decrease of its hypometric trend), all the more pronounced as the subjects were more hypometric in darkness. As a consequence, the post-saccadic retinal error was increased by the Visual Background in the Backward condition and decreased in the Forward condition. Accordingly, the Visual Background also increased the number of S2 and decreased the S2.1 latency in the Backward condition, and led to symmetric changes in the Forward condition.

Fourth, the effect of the Visual Background was to normalize the reaction time of the first secondary saccade among subjects: indeed, in darkness, the more hypometric subjects were, the shorter the latency of their first secondary saccade, whereas with a visual background, the latency of the first secondary saccade tended to become homogenous among subjects.

To our knowledge, the present study provides the first evidence of a visual background influence on the gain of primary reactive saccades. This interesting finding opens a new opportunity to examine saccade behavior in a more complex visual environment in order to determine the contribution of multiples sources of relevant information to saccade generation.

\subsection{Timing of saccade error correction mechanisms}

Our results have shown that corrective saccades depend on post-saccadic retinal error. These results do not favor a strict pre-programming hypothesis, because both the number and accuracy of secondary saccades increase in the medium condition as compared to the brief condition. Furthermore, when the post-saccadic retinal information is present until the execution of the first S2 (medium condition), the final eye position is as accurate as when the target remains visible until the end of the trial (long condition). These findings reinforce previous works supporting the assumption that corrective saccades mostly depend on a retinal error signal (Deubel et al., 1982; Eggert et al., 1999; Prablanc \& Jeannerod, 1974; Prablanc et al., 1978). This is compatible with recent evidence that subjects rely more on visual feedback than on extraretinal signals to generate accurate saccadic corrections in a double step task (Munuera et al., 2009). However, as our experiment in the Dark Background condition revealed a large inter-subject range of primary saccade gain, our data are also compatible with some apparently divergent results and interpretations of the exogenous or endogenous origin of the first secondary saccade (Becker, 1976; Becker \& Fuchs, 1969; Shebilske, 1976). Indeed we observed that when the primary saccade was very hypometric, the large error signal between the short-term target location memory and the saccade efference copy was sufficient to elicit a non-retinal secondary saccade.

\subsection{Effect of visual background on saccade generation and correction mechanisms}

Our results also suggest that saccadic behavior varies according to visual properties of the background. Paradoxically, the presence of a visual background which seemed to reduce target detectability led to an increased final accuracy mainly through a less hypometric primary saccade, irrespective of the jump condition. Note further that, as already mentioned in the previous paragraph, the presence of a visual background has a detrimental effect on the intra-saccadic processing of target location, as expressed by final error in brief stimulus condition. Thus, the visual background may have improved the peripheral processing of static visual signals for S1 planning but at the same time degraded the peri-foveal processing of dynamic (during S1) visual signals to prepare S2.

For stationary targets (fix condition), the presence of a visual background had a beneficial effect on the primary saccade S1. Indeed, as compared to the dark condition, the error at the end of S1 was reduced in the Visual Background condition. A beneficial effect of Visual Background was also noted for the final capture time, an effect which can be related both to a smaller number of secondary saccades and to their slightly smaller reaction time.

As concern the inter-individual variability under the dark condition, the number of S2 was greater for the most hypometric subjects, whereas their S2.1 latencies were smaller, as compared to the corresponding measures for the less hypometric subjects. This observation is in agreement with the known inverse relationship between the latency of S2.1 corrective saccades and their amplitude 
(Becker, 1972). The global effect of the visual background was to homogenize S2.1 latencies between subjects and to equalize the number of secondary saccades for moderate eccentricities.

White et al. (2008) have shown that S1 latency was faster in response to a target presented in a structured or natural background than in a uniform background, when target visibility was matched between background conditions. The authors concluded that the saccadic system compensates for the masking created by structured backgrounds. In our study, we did not find any S1 latency difference, but the following reasons may explain this difference with White et al.'s findings. First, although no detection task was performed, our constant intensity targets presumably had a lower visibility under Visual Background as compared to Dark. It is only when targets visibility was matched (following a detection task performed by the same subjects) that White et al. found faster S1 latencies in the structured background conditions. Second, the fact that White et al. did not report any difference in S1 amplitude could be related to the smaller eccentricity $\left(8^{\circ}\right)$ they used as compared to ours $\left(22.5^{\circ}\right.$ to $37.5^{\circ}$ ). Beutter, Eckstein, and Stone (2003) have already shown that saccades and perception are similarly affected by target contrast. The accuracy of peri-saccadic localization depends on stimulus visibility: indeed, both low luminance stimuli and low contrast stimuli are more mislocalized (Georg, Hamker, \& Lappe, 2008). A third explanation could be the role of attention. Ditterich and colleagues (2000) have tested subjects in detection tasks with two different types of stimuli: one foveal cross target and one circle target much larger than the cross. As subjects' reaction time was smaller with the large circle target, they suggested that subjects' attention was not spatially restricted but widely distributed. In the same vein, Harwood, Madelain, Krauzlis, and Wallman (2008) have shown that the attended region rather than the target size modulates saccadic latencies. In our study with constant size targets, a possible interpretation of the lack of modulation of the saccade latency by the Visual Background is the following. The increased level of subjects' attention led to a trend toward shorter latency of the primary saccade, but at the same time the decreased detectability of the targets from the background led to a trend toward longer latency. These opposite trends may have resulted in the observed similar latency of the primary saccade as in the Dark Background, but with a higher accuracy.

\section{Acknowledgements}

P. Gerardin was supported by an ANR grant to D. Pélisson. The authors thank Christian Urquizar for the data real-time interface and the parameters extraction software.

\section{References}

Bahill, A. T., \& McDonald, J. D. (1983). Frequency limitations and optimal step size for the two-point central difference derivative algorithm with applications to human eye movement data. IEEE Transactions on Biomedical Engineering, 30, 191-194.

Becker, W. (1972). The control of eye movements in the saccadic system. Bibliotheca Opthalmologica, 82, 233-243.

Becker, W. (1976). Do correction saccades depend exclusively on retinal feedback? A note on the possible role of non-retinal feedback. Vision Research, 16, 425-427.

Becker, W., \& Fuchs, A. F. (1969). Further properties of the human saccadic system: Eye movements and correction saccades with and without visual fixation points. Vision Research, 9, 1247-1258.

Beutter, B. R., Eckstein, M. P., \& Stone, L. S. (2003). Saccadic and perceptual performance in visual search tasks. I. Contrast detection and discrimination. Journal of the Optical Society of America A, 20(7), 1341-1355.

Deubel, H., Wolf, W., \& Hauske, G. (1982). Corrective saccades: effect of shifting the saccade goal. Vision Research, 22, 353-364.

Ditterich, J., Eggert, T., \& Straube, A. (2000). The role of the attention focus in the visual information processing underlying saccadic adaptation. Vision Research, 40, 1125-1134.

Eggert, T., Ditterich, J., \& Straube, A. (1999). Intrasaccadic target steps during the deceleration of primary saccades affect the latency of corrective saccades. Experimental Brain Research, 129, 161-166.

Gaveau, V., Pélisson, D., Blangero, A., Urquizar, C., Prablanc, C., Vighetto, A., et al. (2008). Saccade control and eye-hand coordination in optic ataxia. Neuropsychologia, 46, 475-486.

Georg, K., Hamker, F. H., \& Lappe, M. (2008). Influence of adaptation state and stimulus luminance on peri-saccadic localization. Journal of Vision, 8, 1-11.

Greenhouse, D. S., \& Cohn, T. E. (1991). Saccadic suppression and stimulus uncertainty. Journal of the Optical Society of America A, 8, 587-595.

Harwood, M. R., Madelain, L., Krauzlis, R. J., \& Wallman, J. (2008). The spatial scale of attention strongly modulates saccade latencies. Journal of Neurophysiology, 99, 1743-1757.

Henson, D. B. (1978). Corrective saccades: Effects of altering visual feedback. Vision Research, 18, 63-67.

Herman, J. P., Harwood, M. R., \& Wallman, J. (2009). Saccade adaptation specific to visual context. Journal of Neurophysiology, $101,1713-1721$. 
Kapoula, Z., Robinson, D. A., \& Hain, T. C. (1986). Motion of the eye immediately after a saccade. Experimental Brain Research, 61, 386-394.

MacAskill, M. R., Jones, R. D., \& Anderson, T. J. (2003). Saccadic suppression of displacement: effects of illumination and background manipulation. Perception, 32, 463-474.

Matin, E. (1974). Saccadic suppression: A review and an analysis. Psychological Bulletin, 81, 899-917.

Munuera, J., Morel, P., Duhamel, J.-R., \& Deneve, S. (2009). Optimal sensorimotor control in eye movement sequences. Journal of Neuroscience, 29, 3026-3035.

Prablanc, C., \& Jeannerod, M. (1974). Latency and precision of saccades as a function of intensity, duration and retinal position of a stimulus (French). Revue d'Electroencéphalographie et de Neurophysiologie Clinique, 3, 484-488.

Prablanc, C., \& Jeannerod, M. (1975). Corrective saccades: Dependence on reafferent signals. Vision Research, 15, 465-469.

Prablanc, C., Masse, D., \& Echallier, J. F. (1978). Error correcting mechanisms in large saccades. Vision Research, 18, 557-560.

Shebilske, W. L. (1976). Extraretinal information in corrective saccades and inflow vs outflow theories of visual direction constancy. Vision Research, 16, 621-628.

White, B. J., Stritzke, M., \& Gegenfurtner, K. R. (2008). Saccadic facilitation in natural backgrounds. Current Biology, 18, 124-128. 